\title{
Using Institutions for the Study of Qualitative and Quantitative Conditional Logics
}

\author{
Christoph Beierle, Gabriele Kern-Isberner \\ Praktische Informatik VIII - Wissensbasierte Systeme \\ Fachbereich Informatik, FernUniversität Hagen, D-58084 Hagen, Germany
}

\begin{abstract}
It is well known that conditionals need a non-classical environment to be evaluated. In this paper, we present a formalization of conditional logic in the framework of institutions. In regarding both qualitative and probabilistic conditional logic as abstract logical systems, we investigate how they can be related to one another, on the one hand, and to the institution of propositional logic, on the other hand. In spite of substantial differences between these three logics, we find surprisingly clear formal relationships between them.
\end{abstract}

\section{Introduction}

In [6], Goguen and Burstall introduced institutions as a general framework for logical systems. An institution formalizes the informal notion of a logical system, including syntax, semantics, and the relation of satisfaction between them. The latter poses the major requirement for an institution: that the satisfaction relation is consistent under the change of notation. Institutions have also been used as a basis for specification and development languages, see e.g. $[2,13,7]$.

While the examples for institutions in [6] and [7] are based on classical logic, in [1] it is shown that also probabilistic logic can be formalized as an institution. In this paper, we will apply the theory of institutions to conditionals, investigating how the logics of qualitative and probabilistic conditionals fit into that framework. As default rules, conditionals have played a major role in defeasible reasoning (see, e.g., [9]), and assigning probabilities to them opens up a whole universe of possibilities to quantify the (un)certainty of information. It is well-known that conditionals are substantially different from classical logical entities [5]. Nevertheless, we will show that their logic can be formalized as an institution, and thus be compared to classical logical institutions, e.g. that of propositional logic. In detail, we will define an institution of conditional logic, using the semantics of plausibility preorders going back to the works of Lewis [10] and Stalnaker [14], as well as an institution of probabilistic conditionals, using the usual semantics of conditional probabilities.

Now that the logic of conditionals has been given an abstract formal frame, we can also study its relationships to other logics, also being viewed as institutions.

The research reported here was partially supported by the DFG - Deutsche Forschungsgemeinschaft within the CONDOR-project under grant BE 1700/5-1. 
To do so, the formal tool of institution morphisms [6,7] can be used. This will tell us precisely the possibilities how we can interpret e.g. probabilistic conditionals as qualitative conditionals, or in a propositional setting, and vice versa. We will prove that indeed, the institutions of propositional logic, conditional logic and probabilistic conditional logic can be related intuitively, but we will also find that there is essentially exactly one such connection between each pair of these logics. In particular, we will necessarily arrive at a three-valued interpretation of probabilistic conditionals in the propositional framework, reminding us of the three-valued semantics of conditionals [5].

In Sec. 2, we formalize conditional logic as an institution. In Sec. 3, we investigate in detail the relationships between the institutions of conditional logic and of propositional, probabilistic, and probabilistic conditional logic. Section 4 contains some conclusions and points out further work.

\section{Institutions and the Logic of Conditionals}

After recalling the definition of an institution and fixing some basic notation, we first present propositional logic in the institution framework. We then formalize in three steps probabilistic propositional logic, the logic of probabilistic conditionals, and the logic of (qualitative) conditionals as institutions.

\subsection{Preliminaries: Basic Definitions and Notations}

If $C$ is a category, $|C|$ denotes the objects of $C$ and $/ C /$ its morphisms; for both objects $c \in|C|$ and morphisms $\varphi \in / C /$, we also write just $c \in C$ and $\varphi \in C$, respectively. $C^{o p}$ is the opposite category of $C$, with the direction of all morphisms reversed. The composition of two functors $F: C \rightarrow C^{\prime}$ and $G: C^{\prime} \rightarrow$ $C^{\prime \prime}$ is denoted by $G \circ F$ (first apply $F$, then $G$ ). For functors $F, G: C \rightarrow C^{\prime}$, a natural transformation $\eta$ from $F$ to $G$, denoted by $\eta: F \Longrightarrow G$, assigns to each object $c \in|C|$ a morphism $\eta_{c}: F(C) \rightarrow G(C) \in / C^{\prime} /$ such that for every morphism $\varphi: c \rightarrow d \in / C /$ we have $\eta_{d} \circ F(\varphi)=G(\varphi) \circ \eta_{c} . \mathcal{S E \mathcal { T }}$ and $\mathcal{C} \mathcal{A} \mathcal{T}$ denote the categories of sets and of categories, respectively. (For more information about categories, see e.g. [8] or [11].) The central institution definition is the following:

Definition 1. [6] An institution is a quadruple Inst $=\langle\operatorname{Sig}, \operatorname{Mod}, \operatorname{Sen}, \models\rangle$ with a category Sig of signatures as objects, a functor Mod :Sig $\rightarrow \mathcal{C A T}^{\text {op }}$ yielding the category of $\Sigma$-models for each signature $\Sigma$, a functor Sen : Sig $\rightarrow$ $\mathcal{S E \mathcal { T }}$ yielding the sentences over a signature, and a $\mid$ Sig $\mid$-indexed relation $\models_{\Sigma} \subseteq$ $|\operatorname{Mod}(\Sigma)| \times \operatorname{Sen}(\Sigma)$ such that for each signature morphism $\varphi: \Sigma \rightarrow \Sigma^{\prime} \in /$ Sig/, for each $m^{\prime} \in\left|\operatorname{Mod}\left(\Sigma^{\prime}\right)\right|$, and for each $f \in \operatorname{Sen}(\Sigma)$ the following satisfaction condition holds: $\quad m^{\prime} \models_{\Sigma^{\prime}} \operatorname{Sen}(\varphi)(f) \quad$ iff $\operatorname{Mod}(\varphi)\left(m^{\prime}\right) \models_{\Sigma} f$.

For sets $F, G$ of $\Sigma$-sentences and a $\Sigma$-model $m$ we write $m \models_{\Sigma} F$ iff $m \models_{\Sigma} f$ for all $f \in F$. The satisfaction relation is lifted to semantical entailment $\models_{\Sigma}$ between sentences by defining $F \models_{\Sigma} G$ iff for all $\Sigma$-models $m$ with $m=_{\Sigma} F$ we have $m \models_{\Sigma} G . F^{\bullet}=\left\{f \in \operatorname{Sen}(\Sigma) \mid F \models_{\Sigma} f\right\}$ is called the closure of $F$, and $F$ 
is closed if $F=F^{\bullet}$. The closure operator fulfils the closure lemma $\varphi\left(F^{\bullet}\right) \subseteq$ $\varphi(F)^{\bullet}$ and various other nice properties like $\varphi\left(F^{\bullet}\right)^{\bullet}=\varphi(F)^{\bullet}$ or $\left(F^{\bullet} \cup G\right)^{\bullet}=$ $(F \cup G)^{\bullet}$. A consequence of the closure lemma is that entailment is preserved under change of notation carried out by a signature morphism, i.e. $F \models_{\Sigma} G$ implies $\varphi(F) \models_{\varphi(\Sigma)} \varphi(G)$ (but not vice versa).

\subsection{The Institution of Propositional Logic}

In all circumstances, propositional logic seems to be the most basic logic. The components of its institution $\operatorname{Inst}_{\mathcal{B}}=\left\langle\operatorname{Sig}_{\mathcal{B}}, \operatorname{Mod}_{\mathcal{B}}, \operatorname{Sen}_{\mathcal{B}}, \models_{\mathcal{B}}\right\rangle$ will be defined in the following.

Signatures: $\operatorname{Sig}_{\mathcal{B}}$ is the category of propositional signatures. A propositional signature $\Sigma \in\left|\operatorname{Sig}_{\mathcal{B}}\right|$ is a (finite) set of propositional variables, $\Sigma=\left\{a_{1}, a_{2}, \ldots\right\}$. A propositional signature morphism $\varphi: \Sigma \rightarrow \Sigma^{\prime} \in / \operatorname{Sig}_{\mathcal{B}} /$ is a function mapping propositional variables to propositional variables.

Models: For each signature $\Sigma \in \operatorname{Sig}_{\mathcal{B}}, \operatorname{Mod}_{\mathcal{B}}(\Sigma)$ contains the set of all propositional interpretations for $\Sigma$, i.e. $\left|\operatorname{Mod}_{\mathcal{B}}(\Sigma)\right|=\{I \mid I: \Sigma \rightarrow$ Bool $\}$ where Bool $=$ $\{$ true, false $\}$. Due to its simple structure, the only morphisms in $\operatorname{Mod}_{\mathcal{B}}(\Sigma)$ are the identity morphisms. For each signature morphism $\varphi: \Sigma \rightarrow \Sigma^{\prime} \in \operatorname{Sig}_{\mathcal{B}}$, we define the morphism (i.e. the functor in $\left.\mathcal{C} \mathcal{A T}^{o p}\right) \operatorname{Mod}_{\mathcal{B}}(\varphi): \operatorname{Mod}_{\mathcal{B}}\left(\Sigma^{\prime}\right) \rightarrow \operatorname{Mod}_{\mathcal{B}}(\Sigma)$ by $\left(\operatorname{Mod}_{\mathcal{B}}(\varphi)\left(I^{\prime}\right)\right)\left(a_{i}\right):=I^{\prime}\left(\varphi\left(a_{i}\right)\right)$ where $I^{\prime} \in \operatorname{Mod}_{\mathcal{B}}\left(\Sigma^{\prime}\right)$ and $a_{i} \in \Sigma$.

Sentences: For each signature $\Sigma \in S i g_{\mathcal{B}}$, the set $\operatorname{Sen}_{\mathcal{B}}(\Sigma)$ contains the usual propositional formulas constructed from the propositional variables in $\Sigma$ and the logical connectives $\wedge$ (and), $\vee$ (or), and $\neg$ (not). Additionally, the classical (material) implication $A \Rightarrow B$ is used as a syntactic variant for $\neg A \vee B$. The symbols $\top$ and $\perp$ denote a tautology (like $a \vee \neg a$ ) and a contradiction (like $a \wedge \neg a)$, respectively.

For each signature morphism $\varphi: \Sigma \rightarrow \Sigma^{\prime} \in \operatorname{Sig}_{\mathcal{B}}$, the function $\operatorname{Sen}_{\mathcal{B}}(\varphi)$ : $\operatorname{Sen}_{\mathcal{B}}(\Sigma) \rightarrow \operatorname{Sen}_{\mathcal{B}}\left(\Sigma^{\prime}\right)$ is defined by straightforward inductive extension on the structure of the formulas; e.g., $\operatorname{Sen}_{\mathcal{B}}(\varphi)\left(a_{i}\right)=\varphi\left(a_{i}\right)$ and $\operatorname{Sen}_{\mathcal{B}}(\varphi)(A \wedge B)=$ $\operatorname{Sen}_{\mathcal{B}}(\varphi)(A) \wedge \operatorname{Sen}_{\mathcal{B}}(\varphi)(B)$. In the following, we will abbreviate $\operatorname{Sen}_{\mathcal{B}}(\varphi)(A)$ by just writing $\varphi(A)$. In order to simplify notations, we will often replace conjunction by juxtaposition and indicate negation of a formula by barring it, i.e. $A B=A \wedge B$ and $\bar{A}=\neg A$. An atomic formula is a formula consisting of just a propositional variable, a literal is a positive or a negated atomic formula, an elementary conjunction is a conjunction of literals, and a complete conjunction is an elementary conjunction where all atomic formulas appear once, either in positive or in negated form. $\Omega_{\Sigma}$ denotes the set of all complete conjunctions over a signature $\Sigma$; if $\Sigma$ is clear from the context, we may drop the index $\Sigma$. Note that there is an obvious bijection between $\left|\operatorname{Mod}_{\mathcal{B}}(\Sigma)\right|$ and $\Omega_{\Sigma}$, associating with $I \in\left|\operatorname{Mod}_{\mathcal{B}}(\Sigma)\right|$ the complete conjunction $\omega_{I} \in \Omega_{\Sigma}$ in which an atomic formula $a_{i} \in \Sigma$ occurs in positive form iff $I\left(a_{i}\right)=$ true.

Satisfaction relation: For any $\Sigma \in\left|S i g_{\mathcal{B}}\right|$, the satisfaction relation $\models_{\mathcal{B}, \Sigma} \subseteq$ $\left|\operatorname{Mod}_{\mathcal{B}}(\Sigma)\right| \times \operatorname{Sen}_{\mathcal{B}}(\Sigma)$ is defined as expected for propositional logic, e.g. 
$I \models_{\mathcal{B}, \Sigma} a_{i}$ iff $I\left(a_{i}\right)=$ true and $I \models_{\mathcal{B}, \Sigma} A \wedge B$ iff $I \models_{\mathcal{B}, \Sigma} A$ and $I \models_{\mathcal{B}, \Sigma} B$ for $a_{i} \in \Sigma$ and $A, B \in \operatorname{Sen}_{\mathcal{B}}(\Sigma)$.

Proposition 1. Inst $_{\mathcal{B}}=\left\langle\operatorname{Sig}_{\mathcal{B}}, \operatorname{Mod}_{\mathcal{B}}, \operatorname{Sen}_{\mathcal{B}}, \models_{\mathcal{B}}\right\rangle$ is an institution.

Example 1. Let $\Sigma=\{s, t, u\}$ and $\Sigma^{\prime}=\{a, b, c\}$ be two propositional signatures with the atomic propositions $s$ - being a scholar, $t$ - being not married, $u$ - being single and $a$-being a student, $b$ - being young, $c$ - being unmarried. Let $I^{\prime}$ be the $\Sigma^{\prime}$-model with $I^{\prime}(a)=$ true, $I^{\prime}(b)=$ true, $I^{\prime}(c)=$ false. Let $\varphi: \Sigma \rightarrow \Sigma^{\prime} \in \operatorname{Sig}_{\mathcal{B}}$ be the signature morphism with $\varphi(s)=a, \varphi(t)=c, \varphi(u)=c$. The functor $\operatorname{Mod}_{\mathcal{B}}(\varphi)$ takes $I^{\prime}$ to the $\Sigma$-model $I:=\operatorname{Mod}_{\mathcal{B}}(\varphi)\left(I^{\prime}\right)$, yielding $I(s)=I^{\prime}(a)=$ true, $I(t)=I^{\prime}(c)=$ false, $I(u)=I^{\prime}(c)=$ false.

\subsection{The Institution of Probabilistic Propositional Logic}

Based on Inst $_{\mathcal{B}}$, we can now define the institution of probabilistic propositional $\operatorname{logic}$ Inst $_{\mathcal{P}}=\left\langle\operatorname{Sig}_{\mathcal{P}}, \operatorname{Mod}_{\mathcal{P}}, \operatorname{Sen}_{\mathcal{P}}, \models_{\mathcal{P}}\right\rangle$. We will first give a very short introduction to probabilistics as far as it is needed here.

Let $\Sigma \in \mid$ Sig $_{\mathcal{B}} \mid$ be a propositional signature. A probability distribution (or probability function) over $\Sigma$ is a function $P: \operatorname{Sen}_{\mathcal{B}}(\Sigma) \rightarrow[0,1]$ such that $P(\top)=$ 1, $P(\perp)=0$, and $P(A \vee B)=P(A)+P(B)$ for any formulas $A, B \in \operatorname{Sen}_{\mathcal{B}}(\Sigma)$ with $A B=\perp$. Each probability distribution $P$ is determined uniquely by its values on the complete conjunctions $\omega \in \Omega_{\Sigma}$, since $P(A)=\sum_{\omega \in \Omega_{\Sigma}, \omega \models_{\mathcal{B}, \Sigma} A} P(\omega)$. For two propositional formulas $A, B \in \operatorname{Sen}_{\mathcal{B}}(\Sigma)$ with $P(A)>0$, the conditional probability of $B$ given $A$, denoted by $P(B \mid A)$, is $\frac{P(A B)}{P(A)}$. Any subset $\Sigma_{1} \subseteq$ $\Sigma$ gives rise to a distribution $P_{\Sigma_{1}}: \operatorname{Sen}_{\mathcal{B}}\left(\Sigma_{1}\right) \rightarrow[0,1]$ by virtue of defining $P_{\Sigma_{1}}\left(\omega_{1}\right)=\sum_{\omega \in \Omega_{\Sigma}, \omega \models_{\mathcal{B}, \Sigma} \omega_{1}} P(\omega)$ for all $\omega_{1} \in \Omega_{\Sigma_{1}} ; P_{\Sigma_{1}}$ is called the marginal distribution of $P$ on $\Sigma_{1}$.

Signatures: $\mathrm{Sig}_{\mathcal{P}}$ is identical to the category of propositional signatures, i.e. $\operatorname{Sig}_{\mathcal{P}}=\operatorname{Sig}_{\mathcal{B}}$.

Models: For each signature $\Sigma$, the objects of $\operatorname{Mod}_{\mathcal{P}}(\Sigma)$ are probability distributions over the propositional variables, i.e.

$$
\left|\operatorname{Mod}_{\mathcal{P}}(\Sigma)\right|=\{P \mid P \text { is a probability distribution over } \Sigma\}
$$

As for $\operatorname{Mod}_{\mathcal{B}}(\Sigma)$, we assume in this paper that the only morphisms in $\operatorname{Mod}_{\mathcal{P}}(\Sigma)$ are the identity morphisms.

For each signature morphism $\varphi: \Sigma \rightarrow \Sigma^{\prime}$, we define a functor $\operatorname{Mod}_{\mathcal{P}}(\varphi)$ : $\operatorname{Mod}_{\mathcal{P}}\left(\Sigma^{\prime}\right) \rightarrow \operatorname{Mod}_{\mathcal{P}}(\Sigma)$ by mapping each distribution $P^{\prime}$ over $\Sigma^{\prime}$ to a distribution $\operatorname{Mod}_{\mathcal{P}}(\varphi)\left(P^{\prime}\right)$ over $\Sigma . \operatorname{Mod}_{\mathcal{P}}(\varphi)\left(P^{\prime}\right)$ is defined by giving its value for all complete conjunctions over $\Sigma$ :

$$
\left(\operatorname{Mod}_{\mathcal{P}}(\varphi)\left(P^{\prime}\right)\right)(\omega):=P^{\prime}(\varphi(\omega))=\sum_{\omega^{\prime} \models_{\mathcal{B}, \Sigma^{\prime}} \varphi(\omega)} P^{\prime}\left(\omega^{\prime}\right)
$$


where $\omega$ and $\omega^{\prime}$ are complete conjunctions over $\Sigma$ and $\Sigma^{\prime}$, respectively.

Sentences: For each signature $\Sigma$, the set $\operatorname{Sen}_{\mathcal{P}}(\Sigma)$ contains probabilistic facts of the form $A[x]$ where $A \in \operatorname{Sen}_{\mathcal{B}}(\Sigma)$ is a propositional formula from Inst $_{\mathcal{B}} . x \in$ $[0,1]$ is a probability value indicating the degree of certainty for the occurrence of $A$.

For each signature morphism $\varphi: \Sigma \rightarrow \Sigma^{\prime}$, the extension $\operatorname{Sen}_{\mathcal{P}}(\varphi)$ : $\operatorname{Sen}_{\mathcal{P}}(\Sigma) \rightarrow \operatorname{Sen}_{\mathcal{P}}\left(\Sigma^{\prime}\right)$ is defined by $\operatorname{Sen}_{\mathcal{P}}(\varphi)(A[x])=\varphi(A)[x]$.

Satisfaction relation: The satisfaction relation $\models_{\mathcal{P}, \Sigma} \subseteq\left|\operatorname{Mod}_{\mathcal{P}}(\Sigma)\right| \times$ $\operatorname{Sen}_{\mathcal{P}}(\Sigma)$ is defined, for any $\Sigma \in\left|\operatorname{Sig}_{\mathcal{P}}\right|$, by

$$
P \models_{\mathcal{P}, \Sigma} A[x] \quad \text { iff } \quad P(A)=x
$$

Note that, since $P(\bar{A})=1-P(A)$ for each formula $A \in \operatorname{Sen}_{\mathcal{B}}(\Sigma)$, it holds that $P \models_{\mathcal{P}, \Sigma} A[x]$ iff $P \models_{\mathcal{P}, \Sigma} \bar{A}[1-x]$.

Proposition 2. Inst $_{\mathcal{P}}=\left\langle\operatorname{Sig}_{\mathcal{P}}, \operatorname{Mod}_{\mathcal{P}}, \operatorname{Sen}_{\mathcal{P}}, \models_{\mathcal{P}}\right\rangle$ is an institution.

\subsection{The Institution of Probabilistic Conditional Logic}

We now use Inst $_{\mathcal{P}}$ to define the institution of probabilistic conditionals $I_{n} t_{\mathcal{C}}=$ $\left\langle\operatorname{Sig}_{\mathcal{C}}, \operatorname{Mod}_{\mathcal{C}}, \operatorname{Sen}_{\mathcal{C}}, \models_{\mathcal{C}}\right\rangle$.

Signatures: $\operatorname{Sig}_{\mathcal{C}}$ is identical to the category of propositional signatures, i.e. $S i g_{\mathcal{C}}=S i g_{\mathcal{P}}=S i g_{\mathcal{B}}$.

Models: The models for probabilistic conditional logic are again probability distributions over the propositional variables. Therefore, the model functor can be taken directly from probabilistic propositional logic, giving us $\operatorname{Mod}_{\mathcal{C}}=\operatorname{Mod}_{\mathcal{P}}$.

Sentences: For each signature $\Sigma$, the set $\operatorname{Sen}_{\mathcal{C}}(\Sigma)$ contains probabilistic conditionals (sometimes also called probabilistic rules) of the form $(B \mid A)[x]$ where $A, B \in \operatorname{Sen}_{\mathcal{B}}(\Sigma)$ are propositional formulas from Inst $_{\mathcal{B}} . x \in[0,1]$ is a probability value indicating the degree of certainty for the occurrence of $B$ under the condition $A$. - Note that the sentences from Inst $_{\mathcal{P}}$ are included implicitly since a probabilistic fact of the form $B[x]$ can easily be expressed as a conditional $(B \mid T)[x]$ with a tautology as trivial antecedent.

For each signature morphism $\varphi: \Sigma \rightarrow \Sigma^{\prime}$, the extension $\operatorname{Sen}_{\mathcal{C}}(\varphi)$ : $\operatorname{Sen}_{\mathcal{C}}(\Sigma) \rightarrow \operatorname{Sen}_{\mathcal{C}}\left(\Sigma^{\prime}\right)$ is defined by straightforward inductive extension on the structure of the formulas: $\operatorname{Sen}_{\mathcal{C}}(\varphi)((B \mid A)[x])=(\varphi(B) \mid \varphi(A))[x]$.

Satisfaction relation: The satisfaction relation $\models_{\mathcal{C}, \Sigma} \subseteq\left|\operatorname{Mod}_{\mathcal{C}}(\Sigma)\right| \times$ $\operatorname{Sen}_{\mathcal{C}}(\Sigma)$ is defined, for any $\Sigma \in\left|\operatorname{Sig}_{\mathcal{C}}\right|$, by

$$
P \models_{\mathcal{C}, \Sigma}(B \mid A)[x] \quad \text { iff } \quad P(A)>0 \text { and } P(B \mid A)=\frac{P(A B)}{P(A)}=x
$$

Note that for probabilistic facts we have $P \models_{\mathcal{C}, \Sigma}(B \mid \top)[x]$ iff $P(B)=x$ from the definition of the satisfaction relation since $P(T)=1$. Thus, $P \models_{\mathcal{P}, \Sigma} B[x]$ iff $P \models_{\mathcal{C}, \Sigma}(B \mid \top)[x]$.

Proposition 3. Inst $_{\mathcal{C}}=\left\langle\operatorname{Sig}_{\mathcal{C}}, \operatorname{Mod}_{\mathcal{C}}, \operatorname{Sen}_{\mathcal{C}}, \models_{\mathcal{C}}\right\rangle$ is an institution. 


\subsection{The Institution of Conditional Logic}

The institution of conditional logic is $\operatorname{Inst}_{\mathcal{K}}=\left\langle\operatorname{Sig}_{\mathcal{K}}, \operatorname{Mod}_{\mathcal{K}}, \operatorname{Sen}_{\mathcal{K}}, \models_{\mathcal{K}}\right\rangle$ with:

Signatures: $\operatorname{Sig}_{\mathcal{K}}$ is again identical to the category of propositional signatures, i.e. $S i g_{\mathcal{K}}=S i g_{\mathcal{C}}=S i g_{\mathcal{P}}=S i g_{\mathcal{B}}$.

Models: Various types of models have been proposed to interpret conditionals adequately within a formal system (cf. e.g. [12]). Many of them are based on considering possible worlds which can be thought of as being represented by classical logical interpretations $\left|\operatorname{Mod}_{\mathcal{B}}(\Sigma)\right|$, or complete conjunctions $\omega \in \Omega$ (as defined in Sec. 2.2), respectively. One of the most prominent approaches is the system-of-spheres model of Lewis [10] which makes use of a notion of similarity between possible worlds. This idea of comparing worlds and evaluating conditionals with respect to the "nearest" or "best" worlds (which are somehow selected) is common to very many approaches in conditional logics. So, in order to base our conditional logic on quite a general semantics, we take the models to be total preorders over classical propositional interpretations, i.e.

$$
\left|\operatorname{Mod}_{\mathcal{K}}(\Sigma)\right|=\left\{R \mid R \text { is a total preorder on }\left|\operatorname{Mod}_{\mathcal{B}}(\Sigma)\right|\right\}
$$

where a total preorder $R$ is a reflexive and transitive relation such that for any two elements $I_{1}, I_{2}$, we have $\left(I_{1}, I_{2}\right) \in R$ or $\left(I_{2}, I_{1}\right) \in R$ (possibly both).

By identifying $\operatorname{Mod}_{\mathcal{B}}(\Sigma)$ with the set of possible worlds $\Omega$, we will consider the models $R \in \operatorname{Mod}_{\mathcal{K}}(\Sigma)$ to be total preorders on $\Omega$, ordering the possible worlds according to their plausibility. By convention, the least worlds are the most plausible worlds. We will also use the infix notation $\omega_{1} \preceq_{R} \omega_{2}$ instead of $\left(\omega_{1}, \omega_{2}\right) \in R$. As usual, we introduce the $\prec_{R}$-relation by saying that $\omega_{1} \prec_{R} \omega_{2}$ iff $\omega_{1} \preceq_{R} \omega_{2}$ and not $\omega_{2} \preceq_{R} \omega_{1}$. Furthermore, $\omega_{1} \approx_{R} \omega_{2}$ means that both $\omega_{1} \preceq_{R} \omega_{2}$ and $\omega_{2} \preceq_{R} \omega_{1}$ hold.

Each $R \in \operatorname{Mod}_{\mathcal{K}}(\Sigma)$ induces a partitioning $\Omega_{0}, \Omega_{1}, \ldots$ of $\Omega$, such that all worlds in the same partitioning subset are considered equally plausible $\left(\omega_{1} \approx_{R} \omega_{2}\right.$ for $\left.\omega_{1}, \omega_{2} \in \Omega_{j}\right)$, and whenever $\omega_{1} \in \Omega_{i}$ and $\omega_{2} \in \Omega_{k}$ with $i<k$, then $\omega_{1} \prec_{R} \omega_{2}$. Let $\operatorname{Min}(R)$ denote the set of $R$-minimal worlds in $\Omega$, i.e.

$$
\operatorname{Min}(R)=\Omega_{0}=\left\{\omega_{0} \in \Omega \mid \omega_{0} \preceq_{R} \omega \text { for all } \omega \in \Omega\right\}
$$

Each $R \in \operatorname{Mod}_{\mathcal{K}}(\Sigma)$ induces a total preorder on $\operatorname{Sen}_{\mathcal{B}}(\Sigma)$ by setting

$$
\begin{aligned}
& A \preceq_{R} B \quad \text { iff } \quad \text { for all } \omega_{2} \in \Omega \text { with } \omega_{2} \models_{\mathcal{B}, \Sigma} B \\
& \text { there exists } \omega_{1} \in \Omega \text { with } \omega_{1} \models_{\mathcal{B}, \Sigma} A \text { such that } \omega_{1} \preceq_{R} \omega_{2}
\end{aligned}
$$

So, $A$ is considered to be at least as plausible as $B$ (with respect to $R$ ) iff the most plausible worlds satisfying $A$ are at least as plausible as any world satisfying $B$. In particular, if $B \models_{\mathcal{B}, \Sigma} A$, then $A \preceq_{R} B$ for each $R \in \operatorname{Mod}_{\mathcal{K}}(\Sigma)$, since $\omega \models_{\mathcal{B}, \Sigma} B$ implies $\omega \models_{\mathcal{B}, \Sigma} A$. Again, $A \prec_{R} B$ means both $A \preceq_{R} B$ and not $B \preceq_{R} A$. Note that $A \prec_{R} \perp$ for all $A \not \equiv \perp$.

As before, we only consider the identity morphisms in $\operatorname{Mod}_{\mathcal{K}}(\Sigma)$ for this paper. 
For each signature morphism $\varphi: \Sigma \rightarrow \Sigma^{\prime}$, we define a functor $\operatorname{Mod}_{\mathcal{K}}(\varphi)$ : $\operatorname{Mod}_{\mathcal{K}}\left(\Sigma^{\prime}\right) \rightarrow \operatorname{Mod}_{\mathcal{K}}(\Sigma)$ by mapping a (total) preorder $R^{\prime}$ over $\operatorname{Mod}_{\mathcal{B}}\left(\Sigma^{\prime}\right)$ to a (total) preorder $\operatorname{Mod}_{\mathcal{K}}(\varphi)\left(R^{\prime}\right)$ over $\operatorname{Mod}_{\mathcal{B}}(\Sigma)$ in the following way:

$$
\omega_{1} \preceq_{\operatorname{Mod}_{\mathcal{K}}(\varphi)\left(R^{\prime}\right)} \omega_{2} \quad \text { iff } \quad \varphi\left(\omega_{1}\right) \preceq_{R^{\prime}} \varphi\left(\omega_{2}\right)
$$

Note that on the left hand side of (1) the complete conjunctions $\omega_{1}$ and $\omega_{2}$ are viewed as models in $\operatorname{Mod}_{\mathcal{B}}(\Sigma)$, whereas on the right hand side they are sentences in $\operatorname{Sen}_{\mathcal{B}}(\Sigma)$.

It is straightforward to check that $\operatorname{Mod}_{\mathcal{K}}(\varphi)\left(R^{\prime}\right)$ is a total preorder (the corresponding properties are all directly inherited by $R^{\prime}$ ), so indeed $\operatorname{Mod}_{\mathcal{K}}(\varphi)\left(R^{\prime}\right) \in$ $\operatorname{Mod}_{\mathcal{K}}(\Sigma)$. The connection between $R^{\prime}$ and $\operatorname{Mod}_{\mathcal{K}}(\varphi)\left(R^{\prime}\right)$ defined by (1) can also be shown to hold for propositional sentences instead of worlds:

Lemma 1. Let $A, B \in \operatorname{Sen}_{\mathcal{B}}(\Sigma)$. Then $A \preceq_{\operatorname{Mod}_{\mathcal{K}}(\varphi)\left(R^{\prime}\right)} B$ iff $\varphi(A) \preceq_{R^{\prime}} \varphi(B)$.

Corollary 1. Let $A, B \in \operatorname{Sen}_{\mathcal{B}}(\Sigma)$. Then $A \prec_{\operatorname{Mod}_{\mathcal{K}}(\varphi)\left(R^{\prime}\right)} B$ iff $\varphi(A) \prec_{R^{\prime}} \varphi(B)$.

Sentences: For each signature $\Sigma$, the set $\operatorname{Sen}_{\mathcal{K}}(\Sigma)$ contains (propositional) conditionals of the form $(B \mid A)$ where $A, B \in \operatorname{Sen}_{\mathcal{B}}(\Sigma)$ are propositional formulas from $\operatorname{Inst}_{\mathcal{B}}$. For $\varphi: \Sigma \rightarrow \Sigma^{\prime}$, the extension $\operatorname{Sen}_{\mathcal{K}}(\varphi)$ is defined as usual by $\operatorname{Sen}_{\mathcal{K}}(\varphi)((B \mid A))=(\varphi(B) \mid \varphi(A))$.

Satisfaction relation: The satisfaction relation $\models_{\mathcal{K}, \Sigma} \subseteq\left|\operatorname{Mod}_{\mathcal{K}}(\Sigma)\right| \times$ $\operatorname{Sen}_{\mathcal{K}}(\Sigma)$ is defined, for any $\Sigma \in\left|S i g_{\mathcal{K}}\right|$, by

$$
R \models_{\mathcal{K}, \Sigma}(B \mid A) \quad \text { iff } \quad A B \prec_{R} A \bar{B}
$$

Therefore, a conditional $(B \mid A)$ is satisfied (or accepted) by the plausibility preorder $R$ iff its confirmation $A B$ is more plausible than its refutation $A \bar{B}$.

Proposition 4. Inst $_{\mathcal{K}}=\left\langle S i g_{\mathcal{K}}, \operatorname{Mod}_{\mathcal{K}}, \operatorname{Sen}_{\mathcal{K}}, \models_{\mathcal{K}}\right\rangle$ is an institution.

Example 2. We continue our student-example in this qualitative conditional environment, so let $\Sigma, \Sigma^{\prime}, \varphi$ be as defined in Example 1 . Let $R^{\prime}$ be the following total preorder on $\Omega^{\prime}$ :

$$
R^{\prime}: \quad \bar{a} \bar{b} \bar{c} \prec_{R^{\prime}} a b c \approx_{R^{\prime}} \bar{a} b c \prec_{R^{\prime}} a b \bar{c} \approx_{R^{\prime}} a \bar{b} c \approx_{R^{\prime}} a \bar{b} \bar{c} \approx_{R^{\prime}} \bar{a} b \bar{c} \approx_{R^{\prime}} \bar{a} \bar{b} c
$$

Now, for instance $R^{\prime} \models_{\mathcal{K}, \Sigma^{\prime}}(a \mid T)$ since $T \bar{a} \equiv \bar{a}, T \overline{\bar{a}} \equiv a$, and $\bar{a} \prec_{R^{\prime}} a$. Thus, under $R^{\prime}$, it is more plausible to be not a student than to be a student. Furthermore, $R^{\prime} \models_{\mathcal{K}, \Sigma^{\prime}}(c \mid a)$ - students are supposed to be unmarried since under $R^{\prime}, a c$ is more plausible than $a \bar{c}$.

Under $\operatorname{Mod}_{\mathcal{K}}(\varphi), R^{\prime}$ is mapped onto $R=\operatorname{Mod}_{\mathcal{K}}(\varphi)\left(R^{\prime}\right)$ where $R$ is the following total preorder on $\Omega$ :

$$
R: \quad \bar{s} \bar{t} \bar{u} \prec_{R} \bar{s} t u \approx_{R} s t u \prec_{R} s \bar{t} \bar{u} \prec_{R} s t \bar{u} \approx_{R} s \bar{t} u \approx_{R} \bar{s} t \bar{u} \approx_{R} \bar{s} \bar{t} u
$$

As expected, the conditionals $(t \mid s)$ and $(u \mid s)$, both corresponding to $(c \mid a)$ in $\operatorname{Sen}_{\mathcal{K}}\left(\Sigma^{\prime}\right)$ under $\varphi$, are satisfied by $R$ - here, scholars are supposed to be both not married and single. 


\section{Relating Conditional Logic to Other Logics}

Having stepwise developed conditional logic, we now turn to study its interrelationships to the other logics. For instance, there is an obvious translation of sentences mapping $A$ to $(A \mid \top)$ and mapping $(A \mid B)$ to $(A \mid B)[1]$. Furthermore, there is a similar obvious transformation of a propositional interpretation $I$ to a conditional logic model viewing $I$ to be more plausible than any other interpretation, which in turn are considered to be all equally plausible. What happens to satisfaction and entailment when using such translations? In order to make these questions more precise, we use the notion of institution morphisms introduced in [6] (see also [7]).

An institution morphism $\Phi$ expresses a relation between two institutions Inst und Inst' such that the satisfaction condition of Inst may be computed by the satisfaction condition of Inst $^{\prime}$ if we translate it according to $\Phi$. The translation is done by relating every Inst-signature $\Sigma$ to an Inst'-signature $\Sigma^{\prime}$, each $\Sigma^{\prime}$ sentence to a $\Sigma$-sentence, and each $\Sigma$-model to a $\Sigma^{\prime}$-model.

Definition 2. [6] Let Inst $=\langle\operatorname{Sig}, \operatorname{Mod}$, Sen, $\models\rangle$ and Inst $^{\prime}=$ $\left\langle\right.$ Sig $^{\prime}, \operatorname{Mod}^{\prime}$, Sen,$\left.\models^{\prime}\right\rangle$ be two institutions. An institution morphism $\Phi$ from Inst to Inst' is a triple $\langle\phi, \alpha, \beta\rangle$ with a functor $\phi:$ Sig $\rightarrow$ Sig $^{\prime}$, a natural transformation $\alpha:$ Sen $^{\prime} \circ \phi \Longrightarrow$ Sen, and a natural transformation $\beta:$ Mod $\Longrightarrow$ $\operatorname{Mod}^{\prime} \circ \phi$ such that for each $\Sigma \in \mid$ Sig $\mid$, for each $m \in|\operatorname{Mod}(\Sigma)|$, and for each $f^{\prime} \in \operatorname{Sen}^{\prime}(\phi(\Sigma))$ the following satisfaction condition (for institution morphisms) holds: $\quad m \models_{\Sigma} \alpha_{\Sigma}\left(f^{\prime}\right) \quad$ iff $\quad \beta_{\Sigma}(m) \models_{\phi(\Sigma)}^{\prime} f^{\prime}$.

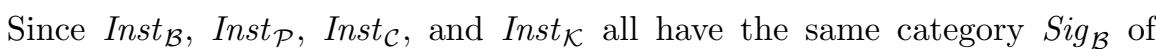
signatures, a natural choice for the signature translation component $\phi$ in any morphism between these institutions is the identity $i d_{S i g_{\mathcal{B}}}$ which we will use in the following.

\subsection{Relating Propositional and Conditional Logic}

The sentences of Inst $_{\mathcal{B}}$ and Inst $_{\mathcal{K}}$ can be related in an intuitive way by sending a propositional formula $A$ to the conditional $(A \mid T)$ having the trivial antecedent $T$. It is easy to check that this translation yields a natural transformations

$$
\alpha_{\mathcal{B} / \mathcal{K}}: \operatorname{Sen}_{\mathcal{B}} \Longrightarrow \operatorname{Sen}_{\mathcal{K}} \quad \alpha_{\mathcal{B} / \mathcal{K}, \Sigma}(A)=(A \mid \top)
$$

Similarly, there is also an intuitive way of mapping a propositional model $I$ to a conditional logic model (which we will denote by $R_{I}$ ). This model $R_{I}$ views $I$ to be more plausible than any other world, and all other worlds are looked upon as equally plausible. With $\omega_{I}$ denoting the unique complete conjunction with $I=_{\mathcal{B}, \Sigma} \omega_{I}$, the preorder $R_{I}$ thus partitions $\Omega$ into the two sets $\left\{\omega_{I}\right\}$ and $\Omega \backslash\left\{\omega_{I}\right\}$. Therfore, $\omega_{I} \prec R_{I} \omega$ for all $\omega \neq \omega_{I}$. Formally, $R_{I}$ is defined by

$$
\omega_{1} \preceq_{R_{I}} \omega_{2} \quad \text { iff } \quad I \models_{\mathcal{B}, \Sigma} \omega_{1} \text { or }\left(I \neq_{\mathcal{B}, \Sigma} \omega_{1} \text { and } I \#_{\mathcal{B}, \Sigma} \omega_{2}\right)
$$

It is straightforward to check that this yields a natural transfromation

$$
\beta_{\mathcal{B} / \mathcal{K}}: \operatorname{Mod}_{\mathcal{B}} \Longrightarrow \operatorname{Mod}_{\mathcal{K}} \quad \beta_{\mathcal{B} / \mathcal{K}, \Sigma}(I)=R_{I}
$$


Having identified obvious standard translations for sentences and models from Inst $_{\mathcal{B}}$ to Inst $_{\mathcal{K}}$, the next question is how to use them in relations between these two institutions. As intuitive as the sentence translation $\alpha_{\mathcal{B} / \mathcal{K}}$ appears, the next proposition shows that it can not be used to define an institution morphism

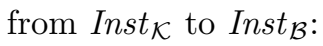

Proposition 5. There is no $\beta$ such that $\left\langle i d_{\text {Sig }_{\mathcal{B}}}, \alpha_{\mathcal{B} / \mathcal{K}}, \beta\right\rangle:$ Inst $_{\mathcal{K}} \longrightarrow$ Inst $_{\mathcal{B}}$ is an institution morphism.

When going in the other direction from Inst $_{\mathcal{B}}$ to Inst $_{\mathcal{K}}$ using the model translation $\beta_{\mathcal{B} / \mathcal{K}}: \operatorname{Mod}_{\mathcal{B}} \Longrightarrow \operatorname{Mod}_{\mathcal{K}}$, we must map conditionals to propositional formulas. A possible choice is to map $(B \mid A)$ to the formula $A B$ confirming the conditional, thereby yielding the natural transformation

$$
\alpha_{\mathcal{K} / \mathcal{B}}: \operatorname{Sen}_{\mathcal{K}} \Longrightarrow \operatorname{Sen}_{\mathcal{B}} \quad \text { with } \quad \alpha_{\mathcal{K} / \mathcal{B}, \Sigma}((B \mid A))=A B
$$

The next proposition shows that $\beta_{\mathcal{B} / \mathcal{K}}$ gives rise to exactly one institution morphism from Inst $_{\mathcal{B}}$ to Inst $_{\mathcal{K}}$, namely the one using $\alpha_{\mathcal{K} / \mathcal{B}}$ for sentence translation:

Proposition 6. $\left\langle i d_{\text {Sig }_{\mathcal{B}}}, \alpha, \beta_{\mathcal{B} / \mathcal{K}}\right\rangle:$ Inst $_{\mathcal{B}} \longrightarrow$ Inst $_{\mathcal{K}}$ is an institution morphism iff $\alpha=\alpha_{\mathcal{K} / \mathcal{B}}$.

\subsection{Relating Conditional and Probabilistic Conditional Logic}

It is quite straightforward to relate the sentences of Inst $_{\mathcal{K}}$ and Inst $_{\mathcal{C}}$ by sending a conditional to a probabilistic conditional with trivial probability 1, yielding the natural transformation

$$
\alpha_{\mathcal{K} / \mathcal{C}}: \operatorname{Sen}_{\mathcal{K}} \Longrightarrow \operatorname{Sen}_{\mathcal{C}} \quad \text { with } \quad \alpha_{\mathcal{K} / \mathcal{C}, \Sigma}((B \mid A))=(B \mid A)[1]
$$

Relating the models of Inst $_{\mathcal{K}}$ and Inst $_{\mathcal{C}}$, however, is far less obvious. Many different ways can be devised to map preorders and probability distributions to one another. As a minimal requirement, we would certainly expect the preorder to be compatible with the ordering induced by the probabilities. As a first approach, we define a mapping sending a probability distribution $P$ to a conditional logic model $R_{P}$. Under $R_{P}$, all complete conjunctions with a positive probability are considered most plausible, and all complete conjunctions with zero probability are taken as less (yet equally) plausible. Thus, $R_{P}$ partitions $\Omega$ into two sets, namely $\operatorname{Min}\left(R_{P}\right)=\{\omega \in \Omega \mid P(\omega)>0\}$ and $\{\omega \in \Omega \mid P(\omega)=0\}$. Formally, $R_{P}$ is defined by

$$
\omega_{1} \preceq R_{P} \omega_{2} \quad \text { iff } \quad P\left(\omega_{2}\right)=0 \text { or }\left(P\left(\omega_{1}\right)>0 \text { and } P\left(\omega_{2}\right)>0\right)
$$

and it is easy to check that this yields a natural transformation

$$
\beta_{\mathcal{C} / \mathcal{K}}: \operatorname{Mod}_{\mathcal{C}} \Longrightarrow \operatorname{Mod}_{\mathcal{K}} \quad \text { with } \quad \beta_{\mathcal{C} / \mathcal{K}, \Sigma}(P)=R_{P}
$$

The next proposition shows that this indeed gives rise to an institution morphism from Inst $_{\mathcal{C}}$ to Inst $_{\mathcal{K}}$ which involves $\alpha_{\mathcal{K} / \mathcal{C}}$.

Proposition 7. $\left\langle i d_{\text {Sig }_{\mathcal{B}}}, \alpha_{\mathcal{K} / \mathcal{C}}, \beta_{\mathcal{C} / \mathcal{K}}\right\rangle:$ Inst $_{\mathcal{C}} \longrightarrow$ Inst $_{\mathcal{K}}$ is an institution morphism. 
What other possibilities are there to generate a preorder from a probability distribution so that the intuitive sentence translation $\alpha_{\mathcal{K} / \mathcal{C}}$ yields an institution morphism? Although the preordering concept would allow a rather fine-grained hierarchy of plausibilities, it is surprising to see that the somewhat simplistic two-level approach of $R_{P}$ is the only possibility to augment $\alpha_{\mathcal{K} / \mathcal{C}}$ towards an institution morphism.

Proposition 8. If $\left\langle i d_{\text {Sig }_{\mathcal{B}}}, \alpha_{\mathcal{K} / \mathcal{C}}, \beta\right\rangle:$ Inst $_{\mathcal{C}} \longrightarrow$ Inst $_{\mathcal{K}}$ is an institution morphism then $\beta=\beta_{\mathcal{C} / \mathcal{K}}$.

Going in the other direction, i.e. from Inst $_{\mathcal{K}}$ to Inst $_{\mathcal{C}}$, we have to transform probabilistic conditionals into qualitative conditionals by a natural transformation $\alpha: S e n_{\mathcal{C}} \Longrightarrow S e n_{\mathcal{K}}$. We might anticipate problems in handling properly nontrivial probabilities, but we would certainly expect that $\alpha_{\Sigma}((B \mid A)[1])=(B \mid A)$. The next proposition, however, shows even this to be impossible.

Proposition 9. There is no institution morphism $\left\langle i d_{\text {Sig }_{\mathcal{B}}}, \alpha, \beta\right\rangle:$ Inst $_{\mathcal{K}} \longrightarrow$ Inst $_{\mathcal{C}}$ such that $\alpha_{\Sigma}((B \mid A)[1])=(B \mid A)$ for all signatures $\Sigma$.

\subsection{Relating Propositional and Probabilistic Conditional Logic}

What is the situation between propositional logic Inst $_{\mathcal{B}}$ and probabilistic conditional logic Inst $_{\mathcal{C}}$ ? Here, the obvious standard translations are

$$
\begin{array}{ll}
\alpha_{\mathcal{B} / \mathcal{C}}: \operatorname{Sen}_{\mathcal{B}} \Longrightarrow \operatorname{Sen}_{\mathcal{C}} & \alpha_{\mathcal{B} / \mathcal{C}, \Sigma}(A)=(A \mid \top)[1] \\
\beta_{\mathcal{B} / \mathcal{C}}: \operatorname{Mod}_{\mathcal{B}} \Longrightarrow \operatorname{Mod}_{\mathcal{C}} & \beta_{\mathcal{B} / \mathcal{C}, \Sigma}(I)=P_{I}
\end{array} \quad P_{I}(\omega)= \begin{cases}1 & \text { if } I(\omega)=\text { true } \\
0 & \text { otherwise }\end{cases}
$$

In [1] it is shown that no institution morphism using the sentence translation $\alpha_{\mathcal{B} / \mathcal{C}}$ exists. When going from propositional logic to probabilistic conditional logic using the model translation $\beta_{\mathcal{B} / \mathcal{C}}$, we have to map probabilistic conditionals to propositional sentences. However, possibly a little surpising at first sight, all probabilistic conditionals with probabilities other than 0 and 1 must be viewed as contradictory propositions.

Proposition 10. If $\left\langle i d_{\text {Sig }_{\mathcal{B}}}, \alpha, \beta_{\mathcal{B} / \mathcal{C}}\right\rangle:$ Inst $_{\mathcal{B}} \longrightarrow$ Inst $_{\mathcal{C}}$ is an institution morphism, then for any $\Sigma \in \operatorname{Sig}_{\mathcal{B}}, \alpha_{\Sigma}$ maps every sentence $(B \mid A)[x]$ with $x \neq 0$ and $x \neq 1$ to $\perp$.

The only choices left for the translation of probabilistic conditionals is thus the translation of conditionals with the trivial probabilities 1 and 0 . Although $(B \mid A)[1]$ represents a conditional if $A$ then $B$ with probability 1 , we can not map it to the classical (material) implication $A \Rightarrow B$ (see [1]). By taking the antecedent as a context into account, we map $(B \mid A)[1]$ to $A \wedge(A \Rightarrow B)$, or equivalently, to $A B$. $(B \mid A)[0]$ is mapped to $\neg(A \Rightarrow B)$, or equivalently, to $A \bar{B}$, since $A \wedge \neg(A \Rightarrow$ $B)=\neg(A \Rightarrow B)$. This yields the natural tranformation

$$
\alpha_{\mathcal{C} / \mathcal{B}}: \operatorname{Sen}_{\mathcal{C}} \Longrightarrow \operatorname{Sen}_{\mathcal{B}} \quad \text { with } \quad \alpha_{\mathcal{C} / \mathcal{B}, \Sigma}((B \mid A)[x])= \begin{cases}A B & \text { if } x=1 \\ A \bar{B} & \text { if } x=0 \\ \perp & \text { otherwise }\end{cases}
$$




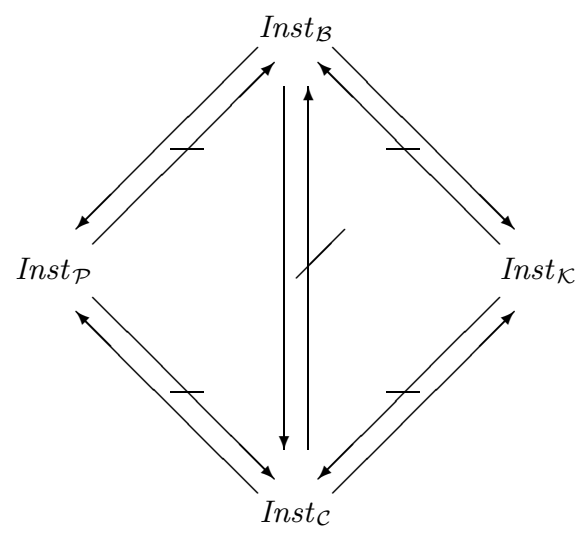

\begin{tabular}{|c|c|c|c|c|}
\hline Morphism & Sent & tence translati & & Model translation \\
\hline Inst $_{\mathcal{B}} \longrightarrow$ Inst $_{\mathcal{P}}$ & $A[x]$ & $\mapsto\left\{\begin{array}{l}\frac{A}{\bar{A}} \\
\perp\end{array}\right.$ & $\begin{array}{l}\text { if } x=1 \\
\text { if } x=0 \\
\text { otherwise }\end{array}$ & $I \mapsto P_{I}$ \\
\hline Inst $_{\mathcal{C}} \longrightarrow$ Inst $_{\mathcal{P}}$ & $A[x]$ & $\mapsto(A \mid \top)[x]$ & & $P \mapsto P$ \\
\hline Inst $_{\mathcal{B}} \longrightarrow$ Inst $_{\mathcal{C}}$ & $(B \mid A)[x]$ & $\mapsto\left\{\begin{array}{l}A B \\
A \bar{B} \\
\perp\end{array}\right.$ & $\begin{array}{l}\text { if } x=1 \\
\text { if } x=0 \\
\text { otherwise }\end{array}$ & $I \mapsto P_{I}$ \\
\hline Inst $_{\mathcal{B}} \longrightarrow$ Inst $_{\mathcal{K}}$ & $(B \mid A)$ & $\mapsto A B$ & & $I \mapsto R_{I}$ \\
\hline Inst $_{\mathcal{C}} \longrightarrow$ Inst $_{\mathcal{K}}$ & $(B \mid A)$ & $\mapsto(B \mid A)[1]$ & & $P \mapsto R_{P}$ \\
\hline
\end{tabular}

Fig. 1. Institution morphisms between Inst $_{\mathcal{B}}$, Inst $_{\mathcal{P}}$, Inst $_{\mathcal{C}}$, and Inst $_{\mathcal{K}}$

Proposition 11. $\left\langle i d_{\operatorname{Sig}_{\mathcal{B}}}, \alpha_{\mathcal{C} / \mathcal{B}}, \beta_{\mathcal{B} / \mathcal{C}}\right\rangle:$ Inst $_{\mathcal{B}} \longrightarrow$ Inst $_{\mathcal{C}}$ is an institution morphism.

Note that $\alpha_{\mathcal{C} / \mathcal{B}, \Sigma}$ reflects the three-valued semantics of conditionals, identifying the verifying part $A B$ and the falsifying part $A \bar{B}$ as most important components of conditional information (cf. $[4,3]$ ).

Figure 1 summarizes our findings with respect to institution morphisms between the four institutions, where the relationships involving Inst $t_{\mathcal{P}}$ are investigated in [1]. Using the intuitive standard translations, we have (essentially) exactly one institution morphism between any pair of the four institutions connected by arrows in Figure 1, but none going in the respective opposite direction. Moreover, the diagram is a commuting one; for instance, $\alpha_{\mathcal{K} / \mathcal{B}}$ is the (vertical) composition of the standard sentence translations $\alpha_{\mathcal{K} / \mathcal{C}}$ and $\alpha_{\mathcal{C} / \mathcal{B}}$, sending $(B \mid A)$ first to $(B \mid A)[1]$ and then to $A B$. Correspondingly, $\beta_{\mathcal{B} / \mathcal{K}}$ is the composition of the standard model translations $\beta_{\mathcal{B} / \mathcal{P}}$ and $\beta_{\mathcal{C} / \mathcal{K}}$, sending $I$ first to $P_{I}$ and then to $R_{I}$. 


\section{Conclusions and Further Work}

In this paper, we used the general framework of institutions to formalize qualitative and probabilistic conditional logic as abstract logical systems. This allowed us to study the structural properties of both syntax and semantics of these logics, telling us, e.g., how conditionals are interpreted under change of notation. Moreover, in making use of the formal vehicle of institution morphisms, we investigated how qualitative and probabilistic conditionals and their respective models can be related to one another, and to the underlying two-valued propositional logic.

For qualitative as well as for probabilistic conditionals, the semantics we based our considerations on are quite standard, in order to make our results most widely applicable. However, there are lots of different semantics for conditionals, and it is an interesting question whether other semantics can yield different relationships between the involved logics. This is a topic of our ongoing research.

\section{References}

1. C. Beierle and G. Kern-Isberner. Looking at probabilistic conditionals from an institutional point of view. In Proceedings of the Workshop Conditionals, Information, and Inference. Hagen, 2002.

2. R. Burstall and J. Goguen. The semantics of Clear, a specification language. In Proceedings of the 1979 Copenhagen Winterschool on Abstract Software Specification, volume 86 of $L N C S$, pages 292-332, Berlin, 1980. Springer-Verlag.

3. P. Calabrese. Deduction and inference using conditional logic and probability. In I. Goodman, M. Gupta, H. Nguyen, and G. Rogers, editors, Conditional Logic in Expert Systems, pages 71-100. Elsevier, North Holland, 1991.

4. B. DeFinetti. Theory of Probability, volume 1,2. John Wiley and Sons, New York, 1974 .

5. D. Dubois and H. Prade. Conditioning, non-monotonic logic and non-standard uncertainty models. In I. Goodman, M. Gupta, H. Nguyen, and G. Rogers, editors, Conditional Logic in Expert Systems, pages 115-158. Elsevier, North Holland, 1991.

6. J. Goguen and R. Burstall. Institutions: Abstract model theory for specification and programming. Journal of the ACM, 39(1):95-146, January 1992.

7. J. A. Goguen and G. Rosu. Institution morphisms. In D. Sannella, editor, Festschrift for Rod Burstall. 2002. (to appear).

8. H. Herrlich and G. E. Strecker. Category theory. Allyn and Bacon, Boston, 1973.

9. G. Kern-Isberner. Conditionals in nonmonotonic reasoning and belief revision. Springer, Lecture Notes in Artificial Intelligence LNAI 2087, 2001.

10. D. Lewis. Counterfactuals. Harvard University Press, Cambridge, Mass., 1973.

11. S. Mac Lane. Categories for the Working Mathematician. Springer-Verlag, New York, 1972.

12. D. Nute. Topics in Conditional Logic. D. Reidel Publishing Company, Dordrecht, Holland, 1980.

13. D. Sannella and A. Tarlecki. Essential comcepts for algebraic specification and program development. Formal Aspects of Computing, 9:229-269, 1997.

14. R. Stalnaker. A theory of conditionals. In N. Rescher, editor, Studies in Logical Theory. American Philosphical Quarterly Monograph Series, No. 2, Blackwell, Oxford, 1968. 